\title{
Multidisciplinarité en conception architecturale : retour sur deux décennies en agence
}

\section{Multidisciplinarity in Architectural Design: A Look on two Decades in Office}

\author{
Charlotte Dautremont ${ }^{1,2, *}$ Vincent Martin $^{2}$ et Sylvie Jancart ${ }^{1}$ \\ ${ }^{1}$ LNA, Faculté d'Architecture, Université de Liège, Belgique \\ ${ }^{2}$ BSolutions, Les Isnes, Belgique
}

\begin{abstract}
Résumé. Ce papier relate vingt années d'expérience d'une agence multidisciplinaire au sein de laquelle le numérique, et particulièrement le BIM, ont permis de décloisonner la conception architecturale et de déployer de nouveaux modèles collaboratifs en architecture. Tout d'abord, nous revenons sur les promesses initiales du numérique en pratique architecturale et les obstacles et raccourcis pris au cours de ces dernières décennies. Nous relatons ensuite l'émergence du nouveau modèle collaboratif multidisciplinaire développé au sein l'agence. En nous basant sur cette expérience de collaboration multidisciplinaire 4.0, nous relatons deux perspectives en cours de développement basées sur le numérique dont le processus collaboratif BIM : la durabilité systémique en conception architecturale d'une part et l'extension de la mission de maîtrise d'œuvre au facility management d'autre part. A travers cet article, nous abordons l'impact du numérique sur la métamorphose de la pratique architecturale à travers la multidisciplinarité collaborative.
\end{abstract}

Mots-clés. Multidisciplinarité, modèles collaboratifs, durabilité, BIM, facility management.

\begin{abstract}
This paper recounts twenty years of experience in a multidisciplinary agency in which digital technology, and particularly BIM, has made it possible to decompartmentalize architectural design and deploy new collaborative models in architecture. Firstly, we look back at the initial promises of digital in architectural practice and the obstacles and shortcuts taken over the past decades. Secondly, we recount the emergence of the new multidisciplinary collaborative model developed within the agency. Based on this experience of multidisciplinary collaboration 4.0, we relate two perspectives under development based on digital technology, including the BIM collaborative process: systemic sustainability in architectural design on the one hand and the extension of the project management mission to facility management on the other hand. Through
\end{abstract}

\footnotetext{
*Corresponding author: cdautremont@uliege.be
} 
this article, we address the impact of digital technology on the metamorphosis of architectural practice through collaborative multidisciplinarity.

Keywords. Multi-disciplinarity, collaborative models, sustainability, BIM, facility management.

\section{Vers l'infini et l'au-delà : décloisonnement}

L'adoption de la digitalisation par les bureaux d'architecture dans les années 90 devait apporter une plus grande cohérence entre la conception architecturale et les décisions structurelles avec pour objectif l'optimisation de la rentabilité. L'ordinateur promettait une évolution fluide à travers les différentes phases d'un projet : une continuité du flux; de l'esquisse à la réalisation bâtie. Le numérique devait offrir par là un champ exploratoire, fait d'une multitude de possibilités en faisant varier toute une série de paramètres, permettant d'atteindre de cette façon une parfaite symbiose, réfléchie et contrôlée, entre forme et structure (Chabard, 2010).

En réalité, dans la majorité des projets, le numérique a balayé ce potentiel. D'un côté, la conception assistée par ordinateur a généralisé la séparation entre forme et structure traduisant ainsi le mouvement du déconstructivisme : "la construction n'explique pas la forme, la forme n'explique pas l'espace et où les lieux sont désintégrés » (D'Alfonso et Samsa, 2002). D'un autre côté, par ces outils numériques, l'œuvre architecturale a évolué vers une stratification extrême, pensée comme une superposition de "layers» (calques « terrain », « structure », « techniques spéciales », etc.) et de peaux (pare-soleil, parement, isolation, structure, finition, etc.) distinctes les unes des autres.

Bien que les outils numériques aient joué un rôle majeur dans cette fragmentation de la conception architecturale, tant à l'échelle macro (le processus collectif de conception) qu'à l'échelle micro (l'objet architectural), il semble difficile d'en décrire distinctement les causes. Est-ce les méthodes traditionnelles de travail qui ont appelé au développement d'une multitude d'outils numériques isolés ou est-ce les outils numériques qui ont accentué nos méthodes segmentées de travail ? Il est fort probable que les outils numériques, ayant répondu aux attentes initiales des concepteurs, n'ont cessé d'évoluer dans ce sens jusqu'à leur paroxysme récent.

Ainsi, opposé aux promesses initiales, l'ordinateur a renforcé le séquençage du flux de travail, le statut «traditionnel» des acteurs et le cloisonnement des compétencesmétiers. En effet, le statut unique de l'architecte, appelé cœur du processus de construction par Hochscheid et Halin (2018a), accentue ce cloisonnement des disciplines. L'architecte conserve sa place centrale dans le processus, autour de laquelle gravitent les autres acteurs. Krygiel et Nies (2008) schématisent la hiérarchisation pyramidale des statuts et traduisent les flux relationnels par des flèches unidirectionnelles (Fig.1). Rasmussen, Gade et Jensen (2017) schématisent à leur tour le flux de travail traditionnel en approche silo (Fig.1.). Les adjectifs et métaphores utilisés pour décrire cette méthode de travail collective traduisent le manque d'interactions et d'échanges qu'elle sous-tend. Malgré la boîte à outils (numériques) performante, le concepteur tend à s'isoler plus que la boîte à outils «ne le rapproche des parties prenantes avec lesquelles il devrait pourtant collaborer », (Celnik et Lebègue, 2015, p.97) 


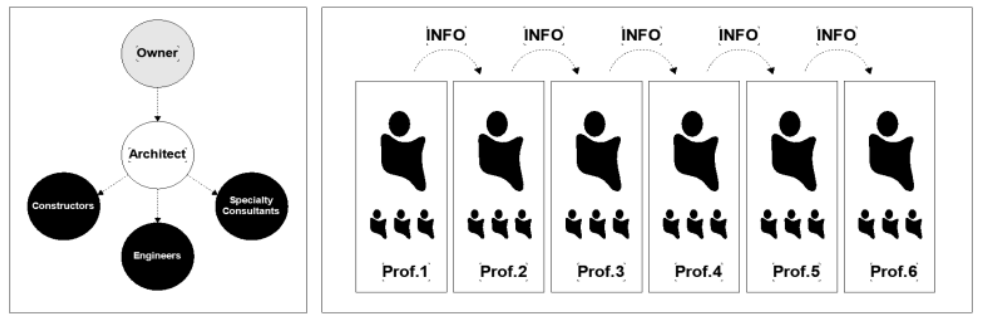

Figure 1. (A gauche) Modèle d'équipe traditionnel sur base de Krygiel et Nies (2008) - (A droite) Approche silo sur base de Rasmussen, Gade et Jensen (2017).

Les conséquences de la rupture du flux de travail sont accentuées par la digitalisation et outrepassent de loin le périmètre bâti du projet. Ce cloisonnement et la stratification organisationnelle ont eu de lourdes répercussions économiques (manque d'efficience de la construction avec surcoûts) (Charef et al., 2019), écologiques (surexploitations des ressources et surproduction de déchets) (Gobbo, 2014; Halbach et Jancart, 2019), entrainant elles-mêmes des conséquences sociétales (santé des intervenants et bien-être des occupants) (Aziz, Nawawi et Ariff, 2016). Si le numérique visait l'optimisation de la rentabilité de travail (Celnik et Lebègue, 2015), seules certaines tâches très spécifiques ont été optimisées. Conscient de ces répercussions, le secteur de la construction a développé le BIM pour y répondre et contrer le manque de rentabilité et de productivité générale du secteur (EU BIM Task Group, 2017).

Cette adoption, d'apparence paradoxale (le BIM, symbole de la construction 4.0, intensifie la digitalisation du secteur), permet pour la première fois de porter toute l'attention sur la collaboration autour du projet, et non plus uniquement sur le projet stricto-senso. Le BIM promet d'atteindre une harmonie entre tous les layers et les strates du projet, tant convoitée dès les prémisses de la numérisation. Il entraine avec lui une refonde en profondeur des pratiques, de l'organisation des compétences-métiers, des rôles, des relations et des responsabilités des acteurs. Cette mutation a pour conséquences que «... les rôles et métiers bien établis tendent à s'interpénétrer, que les phases de projet s'estompent parfois, que de nouveaux modes d'organisation s'imposent et que le travail collaboratif incite à formaliser de nouveaux groupements (...)». Le BIM propose ainsi de «réinterroger les pratiques établies (...)» (Celnik et Lebègue, 2015, p.148-149).

Grâce à ce coup d'œil dans le rétroviseur, il semble réducteur d'aborder la question de la numérisation uniquement à travers le prisme de la conception architecturale et indépendamment des autres compétences du secteur de la construction. Dans ce papier, nous souhaitons aborder la numérisation du secteur à travers la collaboration au sein d'un bureau intégré et de ses développements sur vingt années.

\section{Retour vers le futur}

\subsection{L'union fait la force : multidisciplinarité}

Issue de l'association de deux ingénieurs-architectes et d'un architecte, l'agence est née à l'aube de l'an 2000, en pleine effervescence technologique et principalement en plein développement de la télécommunication. Les associés fondateurs, conscients du potentiel de ce nouveau marché, ont saisi l'occasion pour réunir leurs compétences complémentaires en proposant un travail collaboratif multidisciplinaire. L'agilité technologique d'un des trois associés, traduite par l'utilisation du logiciel STAR ARCHIC dès 1996, a influencé l'éveil et le développement technologique de l'agence. Ce logiciel est resté de nombreuses années un outil pionnier dans la conception architecturale $3 \mathrm{D}$, novateur par ses variantes 
proposées (ARCHI, TOPO et INFRA). Après plusieurs années d'utilisation et bien qu'en accord avec la vision multidisciplinaire de l'agence, l'adoption du logiciel n'a pas été confirmée. Nous comparons cette démarche à l'adoption du BIM étudiée par Hochscheid et Halin (2018b) : les deux facteurs temps et coût (le temps donné à la formation et le retour des connaissance, le coût des licences et du parc informatique requis) ont représenté un effort à fournir important, créant un déséquilibre avec l'utilité perçue au regard des activités et besoins. La décision d'abandon de ce logiciel révèle la difficulté de faire évoluer les compétences et les techniques, simultanément et avec la même intensité. Le choix du logiciel AutoCAD 2DC, rétrograde d'un point de vue des fonctionnalités proposées par rapport au premier, s'est fait suivant un développement de collaboration " humaine ", prioritaire sur le développement technique.

La multidisciplinarité met en lumière l'agence pour de nombreux marchés publics, dont le marché éolien naissant. Ce nouveau marché jalonne à nouveau le développement de l'agence d'une pierre blanche : tout d'abord, un pôle "infrastructure » s'ajoute aux compétences grâce à l'opportunité et la récurrence du marché. Ensuite et surtout, le marché éolien insuffle une prise de conscience de développement durable. Les questions du cycle de vie des projets s'ajoutent aux réflexions sur l'énergie à travers la nécessité d'estomper le déphasage entre conception et exploitation dans les marchés publics. Comment assurer le maintien des compétences du projet ? Comment anticiper les besoins à venir ?

Fort d'une décennie d'existence et d'une collaboration interdisciplinaire efficiente, l'agence étend son dessein à l'architecture durable dès les années 2010 en devenant assesseur BREEAM ${ }^{b}$. Le nouveau siège de l'agence deviendra le faire-valoir de cette nouvelle vision : un bâtiment passif et BREEAM good. Pour atteindre ces performances, des études de simulations dynamiques ont été réalisées : elles ont d'une part appuyé la conception durable du concept architectural initial du projet, tel que l'orientation selon la course du soleil, la compacité et l'implantation semi-enterrée. Mais, d'autre part, ces études ont modifié significativement certains concepts initiaux : à titre d'exemple, la toiture végétalisée s'est transformée en toiture entièrement photovoltaïque avec une autonomie électrique totale du bâtiment. Dans ce projet, la clé de réussite réside majoritairement dans le concours de la multidisciplinarité collaborative, plutôt que dans les outils numériques utilisés.

\subsection{Inévitablement BIM !}

Chacune des décisions de développements collaboratif, constructif et durable, ont conduit inconsciemment au BIM. Celnik et Lebègue (2015) le confirment : "le BIM a été pratiqué ou amorcé sans le savoir par de nombreux professionnels depuis plus de 20 ans. (...) ». L'engagement du BIM coordinateur en 2014 active la progression vers l'efficience collaborative et une nouvelle opportunité de marché. A travers celui-ci, la mission du coordinateur était multiple : adopter REVITC, seul logiciel disponible à offrir une multicompétences BIM et établir des normes de modélisation. Sur le fond et la forme, ce processus a permis une uniformisation à travers des canons graphiques, l'intégration de classifications normalisées et une corrélation avec le cahier des charges. D'abord appliquée au sein des compétences architecture et structure, ce nouveau processus modifie en profondeur les pratiques. En structure, l'étude tridimensionnelle bouleverse la pratique usuelle (passage d'AutoCAD2DC à REVITC). Quant en architecture, l'intégration d'une classification nationale dès les premiers concepts et la rigueur systématique des étapes du projet par la définition des Level of Developments (LOD) ont modifié les habitudes de

\footnotetext{
b BREEAM : certification environnementale des bâtiments, développée par le Building Research Establishment, un organisme privé britannique de recherche.
} 
travail en laissant de côté l'approche « individuelle» empirique de celui-ci. A partir de 2015, les premières maquettes multidisciplinaires émergent (architecture/ structure/ techniques) accompagnées des premières liaisons synchrones entre la maquette numérique, les métrés et cahiers des charges. De part et d'autre, la simultanéité synchronisée de l'étude appuie ce bouleversement : là où traditionnellement l'étude structurelle arrivait en fin de phase avant-projet et celle des techniques spéciales en fin de phase de soumission, le BIM a décloisonné la collaboration des compétences et a amorcé une fluidité dans la collaboration.

Singularité de l'agence, le développement d'une nouvelle compétence est synonyme d'un nouveau défi. Le BIM et son décloisonnement ont permis de répondre à un nouveau marché pour la réalisation de bureaux partagés en coworking pour une maîtrise d'ouvrage anglaise en bouwteam $^{c}$. L'aisance de la maîtrise d'ouvrage vis-à-vis du BIM couplée au programme singulier du projet (rénovation lourde sans modification structurelle) ont permis de travailler à flux tendu entre les étapes d'étude, de prescription et de production avec pour conséquence l'optimisation du processus collaboratif. Cependant, en court-circuitant les étapes d'approbation administratives et de soumission aux entreprises, les phases conventionnelles d'un projet d'architecture ont été éprouvées, et par extension des pratiques architecturales également. De l'optimisation de ces deux types de travail collaboratif (optimisation d'espace de travail pour le coworking et de procédé de travail pour le BIM) résulte une nouvelle durabilité qui pourrait se traduire comme un «anti-gaspillage» de temps, d'espace et de ressources. La durabilité 4.0 est envisageable à deux conditions : une collaboration connectée et la numérisation informée de la conception architecturale. En 2017, l'agence officialise cette combinaison par la création d'une nouvelle cellule en son sein. Nous la décrivons dans le chapitre suivant.

\section{Ginkgo Biloba : résiliente durabilité}

\subsection{Conception durable : de la genèse au cycle de vie}

Construite sur des opportunités de développement, l'évolution de l'agence a été progressive et multidirectionnelle. Les outils numériques ont d'abord rayonné au sein des compétences-métiers architecture et structure pour s'étendre jusqu'aux études en techniques spéciales et infrastructure. Indirectement, l'adoption du BIM en phase conception a profité aux acteurs de la phase construction. Par des boucles d'itération et de validation, la métamorphose numérique de l'agence s'est opérée sur quelques années. Cependant, cette évolution est variable selon les acteurs et la phase du projet.

A travers la Figure 2, nous observons plusieurs constats sur le développement numérique BIM au cours de la phase conception au sein de l'agence. Basé sur le graphe de MacLeamy (2004), nous concentrons notre recherche sur cette phase de conception jalonnée par quatre sous-phases ${ }^{\mathrm{d}}$ de délivrables BIM : un projet modélisé, informé et documenté selon un niveau de détail propre à son avancement (LoD). Ces jalons sont aussi des sasses de validation qui s'accompagnent de boucles d'itération (boucle en traits tillés sur la Figure 2). Ces boucles permettent un développement à l'échelle du projet et à l'échelle du développement numérique et collaboratif de l'agence. Les acteurs (stakeholders by skills sur la Figure 2) interviennent, de plus en plus nombreux au fil de

\footnotetext{
${ }^{\mathrm{c}}$ Bouwteam : la collaboration entre la maîtrise d'ouvrage, l'équipe de concepteurs et le constructeur, travaillant ensemble, dès la genèse du projet jusqu'à sa construction.

d Les quatre sous-phases : esquisse (pre-design) au LoD100, avant-projet simplifié (design concept) au LoD200, avant-projet détaillé et dossier administratif (detailling \& engineering) au LoD300 et dossier d'exécution (construction documentation) au LoD350.
} 
l'avancée du projet et avec eux un niveau de compétences traduit dans le niveaux de détails des LoD.

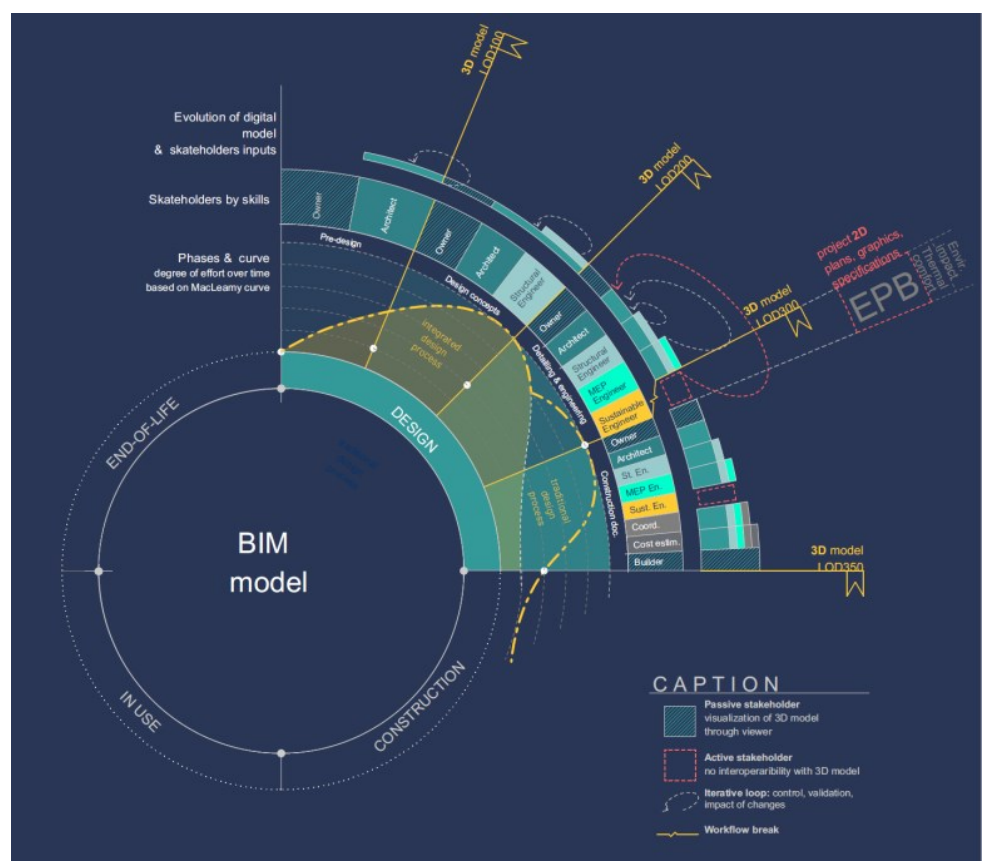

Figure 2. Flux de travail collaboratif et rupture en phase conception, basé sur le graphe de MacLeamy.

Suivant la courbe de MacLeamy, nous pouvons estimer le processus collaboratif BIM par l'effort fourni au sein des phases de développement et l'impact coût sur le projet. Dans un processus BIM, l'effort culmine en phase d'avant-projet, avec pour objectif, toujours selon MacLeamy, une diminution de l'effort en fin de phase avant-projet détaillé (APD) et des bénéfices en phase dossier d'exécution (DE).

Cependant, notre étude au sein de l'agence a mis en lumière une rupture du flux de travail en fin de phase APD dû entre autres à l'étude tardive de la durabilité (sustainable engineer sur la Figure.2) du projet d'une part (LoD300), et d'autre part, dû à un manque d'interopérabilité des outils numériques disponibles pour cette étude durable. En effet, lors du développement du processus BIM à toutes les compétences métiers, le choix des outils numériques s'est porté sur des logiciels efficients d'un point de vue des études durables, tels que DesignBuilder(C) pour les études thermodynamiques, mais dont l'interopérabilité n'était, et n'est toujours pas, performante. Ce choix s'est fait avec l'espoir d'un développement rapide d'interopérabilité du logiciel tout en privilégiant la qualité de l'outil métier. Ce manque d'interopérabilité entraîne encore aujourd'hui une conversion en 2D du projet BIM, une re-modélisation et souvent des pertes d'informations. Cette rupture de flux provoque des boucles d'itération très importantes (validations, variantes, changements, etc.) et donc une perte des bénéfices de l'effort initial relevé dans la courbe de MacLeamy. Cette situation se traduit, non pas par le choix entre la courbe du processus traditionnel de conception ou la courbe du processus de conception intégré, mais par la réunion des deux courbes avec un effort intense dès la phase APD jusqu'en DE (courbe en gras et en traits tillés / pointillés, Fig. 2).

Se basant sur ce constat, le développement d'une conception durable et collaborative est devenu l'un des nouveaux axes de développement de l'agence en 2019. Ce 
développement, BIM et durabilité, jalonne les vingt années d'existence de l'agence avec deux nouvelles perspectives explicitées dans les deux prochaines sous sections.

\subsection{A la recherche d'une méthode collaborative, systémique et numérique}

La première perspective vise l'intégration d'une durabilité systémique dès les prémices de la conception architecturale, grâce au processus BIM. A cette fin, nous proposons d'intégrer l'étude de la durabilité dès la phase esquisse par l'ingénieur durable (Sust. En, Fig.3). De cette manière, la courbe d'effort reprend l'allure proposée par MacLeamy et annihile la rupture en fin de phase APD avec des boucles d'itération plus petites comme illustré en Figure 3. L'objectif de notre recherche est le développement d'une méthode de travail collaborative profitant des avantages des outils numériques tels que le BIM autour de l'avatar numérique. Cette recherche est actuellement en cours et fera l'objet d'autres publications à venir.

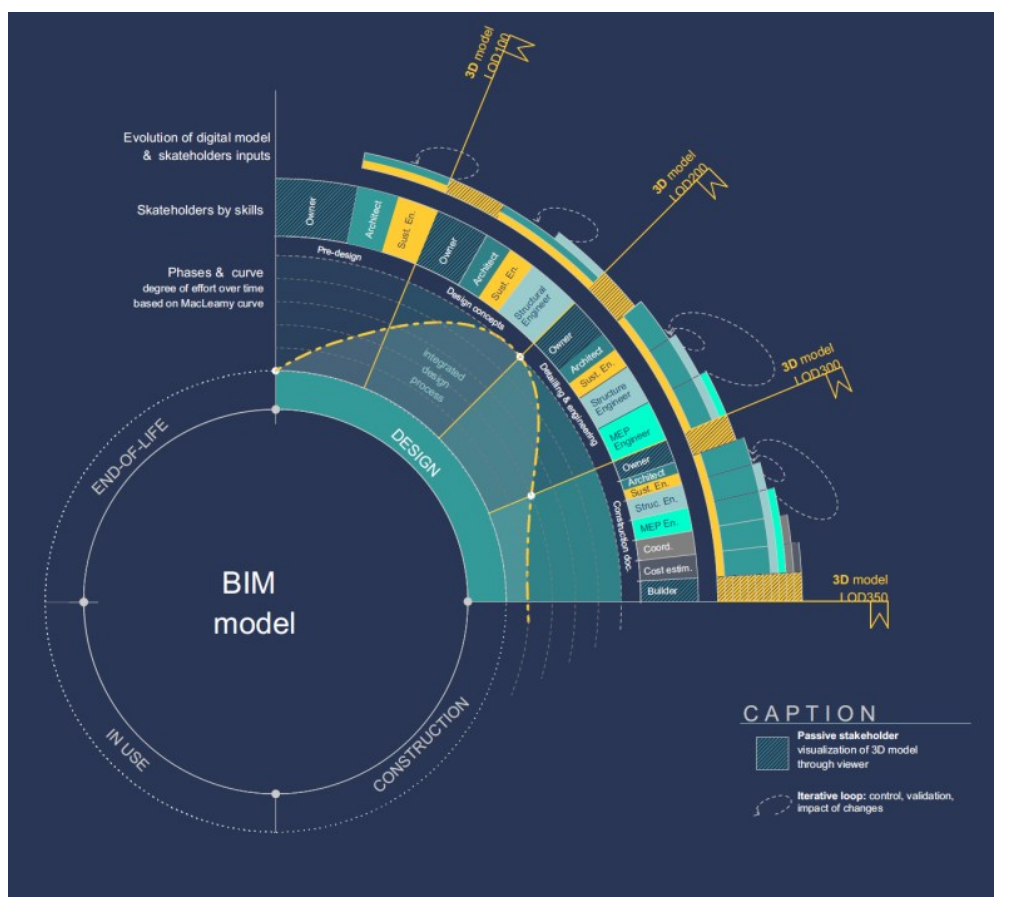

Figure 3. Intégration d'une durabilité dès l'esquisse de la conception architecturale par la collaboration - Modification de la courbe d'effort.

\subsection{Métamorphose de la pratique architecturale: $7 \%$ contre $73 \%$}

Le cycle de vie d'un bâtiment est un concept peu abordé ou même anticipé dans la conception architecturale. Empiriquement, les concepteurs; architectes, ingénieurs et constructeurs continuaient une histoire déjà écrite (projet de rénovation) ou partaient d'une page blanche (projet neuf), sans projection future particulière. Pourtant, les phases conception - construction réunies, représentent à elles deux environ $7 \%$ du coût du cycle de vie d'un bâtiment contre $73 \%$ pour la phase maintenance et utilisation (Aziz, Nawawi et

e Nous entendons par durabilité systémique un ensemble complexe et évolutif de principes durables interdépendants où les principes sont des sous-ensembles. 
Ariff, 2016). Consciente de cette réalité et parallèlement à la recherche axée sur la phase conception architecturale durable précitée, l'agence développe actuellement une nouvelle mission au service de la maintenance du projet (Facility Mangement, FM).

La nouvelle mission de maintenance du projet se développe en deux phases.

Tout d'abord, l'agence étant habituée à collaborer avec d'autres compétences-métiers en interne a récemment proposer d'étendre sa mission multidisciplinaire de conception à du FM. Grâce à ses acquis numériques collaboratifs, la mission de FM a pour objectif d'assurer la performance énergétique d'un bâtiment tertiaire basse énergie. Cette mission FM est réservée dans un premier temps à l'équipe de conception technique et durable.

Rapidement, la mission a relevé des inconnues, et ce malgré la bonne connaissance du projet en tant que maîtrise d'œuvre. Ces inconnues sont liées à la double évolution du projet sur son cycle de vie : d'un côté, l'évolution physique du bâti tels que les transformations, et de l'autre, l'évolution usuelle du bâtiment, impondérable.

Dans la première évolution, les changements physiques sont facilement abordables avec l'avatar numérique BIM as-built, à l'inverse de l'évolution usuelle. Malgré des équipements techniques précis et une gestion technique centralisée (GTC), on remarque que certaines données sont difficilement appréhendables, tels que et à titre d'exemple l'humidité, la qualité intérieure de l'air, la luminosité et le bruit.

Sur base de ces constats, l'agence développe la seconde phase de la mission de maintenance du projet : lier la maquette numérique BIM as-built du projet aux installations mises en place (capteurs et gestion technique centralisée GTC). Ce développement FM comporte de manière plus subtile une notion de durabilité en garantissant le maintien des objectifs fixés.

Dans ce projet, le processus collaboratif BIM et la maquette numérique $(\mathrm{MN})$, permettront à termes de modifier les conventions contractuelles classiques d'une mission architecturale d'une maîtrise d'œuvre en étirant la démarche durable au cycle de vie du projet. Ce projet toujours en cours de développement fera l'objet de publications futures.

\section{Conclusion}

Dans ce papier, nous sommes revenus sur les aspirations initiales du numérique en architecture et des travers développés au fil des années, dont la stratification de l'objet architectural et la segmentation du travail collectif. A la lumière de ces constats, nous avons abordé notre recherche à travers le prisme de la collaboration et de la multidisciplinarité tel que le processus BIM. S'appuyant sur l'expérience d'une agence multidisciplinaire de vingt années d'expériences, cette approche démontre qu'au sein de l'agence, le numérique a renforcé les fondations existantes (multidisciplinarité grâce au BIM) et permet aujourd'hui l'essor de nouveaux modèles de conception architecturale tels que l'intégration d'une durabilité systémique dès l'esquisse.

En nous basant sur les avantages du processus collaboratif au sein et à travers plusieurs disciplines de l'agence, nous avons relevé les conséquences d'un développement partiel du processus, c'est-à-dire non appliqué à l'ensemble multidisciplinaire. Sur base de ces constats, nous avons esquissé les opportunités qu'apporte le numérique dans l'intégration d'une durabilité systémique en conception architecturale.

Parallèlement à l'intégration de durabilité, l'article relate succinctement le développement en cours et futur de l'agence. L'extension de mission au cours du cycle de vie du projet est le nouvel objectif de l'agence : étendre la mission de conception architecturale à la gestion patrimoniale grâce au numérique tels que le BIM as-built.

Ainsi, nous remarquons que durant ces deux décennies, l'agence a profité du développement numérique, connecté et collaboratif, pour faire évoluer sa pratique de maîtrise d'œuvre tout en restant ancrée dans son objectif initial de multidisciplinarité. 
A la lumière de ces faits, il nous semble opportun d'espérer l'émergence de nouveaux modèles collaboratifs, complexes et systémiques, permettant de répondre aux futurs enjeux sociétaux et environnementaux.

\section{Remerciements}

Les auteurs remercient les collaborateurs de l'agence et particulièrement l'Architecte fondateur pour le partage de ses mémoires, ainsi que l'ensemble de la direction de soutenir ce projet de recherche. Cet article fait partie d'une recherche de thèse en entreprise (recherche financée en partie par la cellule d'Aides à la Recherche et à l'Innovation Technologique du Service Public de Wallonie), dont certaines parties seront traitées plus en profondeur ultérieurement.

\section{Bibliographie}

Aziz, N. D., Nawawi, A. H. and Ariff, N. R. M. (2016) 'Building Information Modelling (BIM) in Facilities Management: Opportunities to be Considered by Facility Managers', Procedia - Social and Behavioral Sciences. doi: 10.1016/j.sbspro.2016.10.252.

Celnik, O. and Lebègue, E. (2015) BIM et maquette numérique: pour l'architecture, le bâtiment et la construction. Deuxième. Edited by Eyrolles.

Chabard, P. (2010) 'Antoine Picon. Culture numérique et architecture : une introduction', Critique d'art. Actualité internationale de la littérature critique sur l'art contemporain.

Charef, R. et al. (2019) 'Building Information Modelling adoption in the European Union: An overview', Journal of Building Engineering. doi: 10.1016/j.jobe.2019.100777.

D'Alfonso, E. and Samsa, D. (2002) L'architecture, les formes et les styles de l'Antiquité à nos jours. Edited by Solar (2000).

EU BIM Task Group (2017) Handbook for the introduction of Building Information Modelling by the European Public Sector Strategic action for construction sector performance: driving value, innovation and growth, EUBIM Task Group.

Gobbo, E. (2014) Déchets de construction, matières à conception. Analyse des stocks et flux de matières dans le cadre des opérations de rénovation énergétique en Région de Bruxelles-Capitale. Université Catholique de Louvain UCL.

Halbach, A. and Jancart, S. (2019) 'BUILDING FOR RESOURCE RECOVERY THROUGH BIM, Interviews with practicing architects', Responsive Cities, disrupting through circular design, IAAC, pp. 1-8.

Hochscheid, E. and Halin, G. (2018a) 'BIM implementation in architecture firms: interviews, case studies and action research used to build a method that facilitates implementation of BIM implementation of BIM', eCAADe 2018 36th Annual Conference.

Hochscheid, É. and Halin, G. (2018b) 'L'adoption du BIM dans les agences d'architecture en France', SHS Web of Conferences. doi: 10.1051/shsconf/20184701009.

Krygiel, E. and Nies, B. (2008) Green BIM : Successful sustainable design with building information modeling, Indianalolis: Wiley Publishing, IN.

MacLeamy, P. 2004. MacLeamy curve, in Collaboration, Integrated Information, and the Project Lifecycle in Building Design and Construction and Operation (WP-1202). CURT, August, 2004

Rasmussen, M. B., Gade, A. N. and Jensen, R. L. (2017) 'A Knotworking Guideline for Building Projects: Bridging the Gap between Participants', International Journal of Engineering Research and Applications. doi: 10.9790/9622-0706042440. 